\title{
Logarithmic Stability for Coefficients Inverse Problem of Coupled Schrödinger Equations
}

\author{
Fangfang Dou* and Masahiro Yamamoto ${ }^{\dagger \ddagger}$
}

\begin{abstract}
In this paper, we study an inverse coefficients problem for two coupled Schrödinger equations with an observation of one component of the solution. The observation is done in a nonempty open subset of the domain where the equations hold. A logarithmic type stability result is obtained. The main method is based on the Carleman estimate for coupled Schrödinger equations and coupled heat equations, and the Fourier-BrosIagolnitzer transform.
\end{abstract}

Keywords: logarithmic stability, coefficients inverse problem, coupled Schrödinger equations, Carleman estimate

\section{Introduction}

Let $T>0$ and $\Omega \subset \mathbb{R}^{3}$ be a nonempty bounded domain with smooth boundary and let $i=\sqrt{-1}$. Consider the following coupled Schrödinger equations:

$$
\begin{cases}i \partial_{t} y_{1}+\Delta y_{1}+a_{11}(x) y_{1}+a_{12}(x) y_{2}=0 & \text { in } \Omega \times(0, T), \\ i \partial_{t} y_{2}+\Delta y_{2}+a_{21}(x) y_{1}+a_{22}(x) y_{2}=0 & \text { in } \Omega \times(0, T), \\ y_{1}=0, y_{2}=0 & \text { on } \Gamma \times(0, T), \\ y_{1}(x, 0)=y_{10}, y_{2}(x, 0)=y_{20} & \text { in } \Omega .\end{cases}
$$

System (1) is a useful model for describing molecular multiphoton transitions induced by a laser (e.g. [1,12]), where $a_{11}(x)$ and $a_{22}(x)$ are field-free molecular electronic potentials, and $a_{12}(x)$ and $a_{21}(x)$ are radiation-molecule interactions. In physical models, usually,

\footnotetext{
${ }^{*}$ School of Mathematical Sciences, University of Electronic Science and Technology of China, Chengdu, China. Email: fangfdou@uestc.edu.cn.

${ }^{\dagger}$ Department of Mathematical Sciences, The University of Tokyo, 3-8-1 Komaba, Meguro, Tokyo 153, Japan. Email: myama@ms.u-tokyo.ac.jp.

${ }^{\ddagger}$ PeoplesFriendship University of Russia (RUDN University), 6 Miklukho-Maklaya St, Moscow, 117198, Russian Federation
} 
the radiation-molecule interactions can be deduced a priori while the field-free molecular electronic potentials should be determined a posteriori.

Let $\omega$ be a nonempty open subset of $\Omega$. In this paper, we study the following inverse problems:

Problem (IP) Can one recover the field-free molecular electronic potentials $\left(a_{11}, a_{22}\right)$ from suitable observation of $y_{1}$ on $[0, T] \times \omega$ ?

Here the word "recover" means two issues: One is that the observation determines the potentials uniquely. The other is to find an algorithm to compute the potentials efficiently.

A stability estimate

$$
\left\|\left(a_{11}, a_{22}\right)\right\| \leq C\left\|\left.y_{1}\right|_{\omega}\right\|
$$

with suitable norms under suitable boundedness conditions is not only important theoretically but also essential for the second issue: it can guarantee the convergence of the numerical algorithm for computing $\left(a_{11}, a_{22}\right)$.

Inequalities in the type of (2) for Schrödinger equations were studied extensively (e.g. $[2-5,7,8,10,14,17,19])$. Roughly speaking, the existing works fall into two categories: one is Lipschitz type stability when the observation domain fulfills some geometrically condition (e.g. $[2,3,7,8,10,14,17,19]$ ), while the other is logarithmic type stability when the observation domain is a general nonempty open subset of the domain or its boundary (e.g. $[4,5]$ ). For the latter case, some a priori knowledge about the potential on a suitable subdomain should be known (see [5]).

A main method for establishing the Lipschitz type stability is based on Carleman estimate. On the other hand, the key method for proving the logarithmic type stability is a combination of the Carleman estimate and the Fourier-Bros-Iagolnitzer (F.B.I.) transformation. For readers who are not familiar with the F.B.I. transform, we refer them to [9] for an introduction and to [18] for the application of F.B.I. transform to establish observability estimate for Schrödinger equations.

To the best of our knowledge, although there are several interesting works concerning inverse problem for a parabolic system with two components by measurements of one component, for [6] as an example, there is no work on the inverse coefficients problem for the coupled Schrödinger equations with an observation on one component of the solution. Due to the essential difference between these two equations, we have to argue independently of [6] in the case of parabolic systems. In this paper, we will study this problem by the Carleman estimate for Schrödinger equation, coupled heat equations and F.B.I. transform. Although we borrow some idea in [5] to prove our main result, since we study the inverse problem for couple Schrödinger equations with a single observation on one component of the solution, we cannot simply mimic the method in [5] to obtain the desired logarithmic type stability. Some technical obstacles should be overcome, as is seen in the proof.

The rest of this paper is organized as follows. Section 2 is devoted to presenting the main result while section 3 is devoted to the proof of the main result. 


\section{Statement of the main result}

Let $\omega_{0}$ be an open subset of $\Omega$ such that there exists a function $\phi \in C^{4}(\bar{\Omega})$ satisfying

$$
\left\{\begin{array}{l}
\nabla \phi \neq 0 \text { in } \overline{\Omega \backslash \omega_{0}}, \\
\frac{\partial \phi}{\partial \nu} \leq 0 \text { on } \partial \Omega, \\
|\nabla \phi(x) \cdot \xi|^{2}+\sum_{i, j=1}^{3}\left(\partial_{j} \partial_{j} \phi(x)\right) \xi_{j} \xi_{j}>0 \quad \text { in } \overline{\Omega \backslash \omega_{0}}, \forall \xi=\left(\xi_{1}, \xi_{2}, \xi_{3}\right) \in \mathbb{R}^{3}, \\
\phi(x)>\frac{2}{3}\|\phi\|_{L^{\infty}(\Omega)}, \quad \forall x \in \Omega .
\end{array}\right.
$$

Here $\nu=\nu(x)$ denotes the outward normal vector of $\Omega$.

There are plenty of choices of $\omega_{0}$ satisfying the above condition. A typical example can be constructed as follows.

Let $x_{0} \in \mathbb{R}^{3} \backslash \bar{\Omega}$ and

$$
\Gamma_{0} \triangleq\left\{x \in \Gamma \mid\left(x-x_{0}\right) \cdot \nu(x) \geq 0\right\}
$$

Let $\delta>0$. Put

$$
\omega_{0}=O_{\delta} \triangleq\left\{x \in \Omega \mid \operatorname{dist}\left(x, \Gamma_{0}\right)<\delta\right\} .
$$

Let $\tilde{\psi}(\cdot) \in C^{4}(\bar{\Omega})$ be a nonnegative function such that $\tilde{\psi}(x)=\left|x-x_{0}\right|^{2}$ for $x \in \overline{\Omega \backslash \omega_{0}}$ and $\tilde{\psi}(x)>0$ for $x \in O_{\delta / 2}$ and $\tilde{\psi}=0$ on $\Gamma_{0}$. Then $\psi(x)=\tilde{\psi}(x)+2|\tilde{\psi}|_{L^{\infty}(\Omega)}$ is the desired function.

More examples of such kind of $\omega_{0}$ and $\psi$ can be found in [17].

Clearly, if (3) holds, then there exists $\omega_{1} \subset \subset \omega_{0}$ such that

$$
\left\{\begin{array}{l}
\nabla \phi \neq 0 \text { in } \overline{\Omega \backslash \omega_{1}}, \\
|\nabla \phi(x) \cdot \xi|^{2}+\sum_{i, j=1}^{3}\left(\partial_{j} \partial_{j} \phi(x)\right) \xi_{j} \xi_{j}>0 \quad \text { in } \overline{\Omega \backslash \omega_{1}}, \forall \xi=\left(\xi_{1}, \xi_{2}, \xi_{3}\right) \in \mathbb{R}^{3} .
\end{array}\right.
$$

Let $\tilde{\omega} \in \Omega$ be a neighborhood of $\omega_{1}$ such that $\omega_{1} \subset \subset \tilde{\omega}$ and $\partial \tilde{\omega}$ is $C^{2}$. Set

$$
\mathcal{H}=C^{1}\left(0, T ; H^{2}(\Omega)\right) \cap C^{2}\left(0, T ; H^{1}(\Omega)\right),
$$

where $H^{k}(\Omega)$ is the usual Sobolev space. The Banach space $\mathcal{H}$ is equipped with its natural norm

$$
\|z\|_{\mathcal{H}}^{2}=\|z\|_{C^{1}\left(0, T ; H^{2}(\Omega)\right)}^{2}+\|z\|_{C^{2}\left(0, T ; H^{1}(\Omega)\right)}^{2}, \quad \forall z \in \mathcal{H}
$$

Let $\omega \subset \omega_{1} \subset \Omega$ be an arbitrary nonempty open subset. Suppose that $\left\{a_{j k}\right\}_{j, k=1}^{2} \subset L^{\infty}(Q)$ and we can choose a constant $a_{0}>0$ such that

$$
a_{21} \geq a_{0} \text { or }-a_{21} \geq a_{0} \text { in } \omega \times(0, T) .
$$


Remark 2.1. (7) means that the coupling between $y_{1}$ and $y_{2}$ does not degenerate. More precisely, $y_{1}$ can effect $y_{2}$ adequately. Without (7), one cannot obtain information of $y_{2}$ from $y_{1}$.

Let us now define the admissible set of unknown coefficients. Fix a constant $M>0$ and two functions $\varpi_{1}, \varpi_{2} \in L^{\infty}(\tilde{\omega} ; \mathbb{R})$. Let $\mathcal{A}(\tilde{\omega}, M)$ be the set of pairs of real-valued functions $\left(a_{11}, a_{22}\right)$ such that

$$
\begin{aligned}
\mathcal{A}(\tilde{\omega}, M) \triangleq & \left\{\left(a_{11}, a_{22}\right) \in L^{\infty}(\Omega ; \mathbb{R})^{2} \mid\left\|a_{j j}\right\|_{L^{\infty}(\Omega)} \leq M, a_{j j}(x)=\varpi_{j}(x) \text { on } \tilde{\omega},\right. \\
& \text { the equation }(1) \text { has a unique solution }\left(y_{1}, y_{2}\right) \in \mathcal{H} \text { satisfying } \\
& \left.\left\|y_{j}\right\|_{\mathcal{H}} \leq \mathbf{C}(M) \text { for some constant } \mathbf{C}(M) \text { depending on } M, j=1,2\right\} .
\end{aligned}
$$

Remark 2.2. There are mainly two restrictions on a element in $\mathcal{A}(\tilde{\omega}, M)$. The first one is that there is a priori bound $M$. This is reasonable since in a physical model, one can assume to know some preliminary upper bound on unknown potentials. The second one is that we know the value of $\left(a_{11}(x), a_{22}(x)\right)$ for $x \in \tilde{\omega}$. This is technically restrictive but is acceptable because we may be able to directly measure potentials near the boundary. Furthermore we note that compared with [5], we need less information on unknown potentials.

In what follows, in order to emphasize the dependence of the solution to (1) on the unknown potentials, we write $\left(y_{1}\left(a_{11}, a_{22}\right), y_{2}\left(a_{11}, a_{22}\right)\right)$ for the solution to (1).

We choose the initial data $\left(y_{10}, y_{20}\right)$ which satisfy all conditions ensuring that $\mathcal{A}(\tilde{\omega}, M)$ is nonempty. Also, for $j=1,2$, they fulfill

$$
\left\{\begin{array}{l}
y_{j 0}(x) \in \mathbb{R} \text { or } i y_{j 0}(x) \in \mathbb{R} \text { a.e. in } \Omega \\
\left|y_{j 0}(x)\right| \geq r>0 \text { a.e. in } \Omega \\
y_{j}\left(a_{11}, a_{22}\right) \in H^{1}\left(0, T ; L^{\infty}(\Omega)\right) .
\end{array}\right.
$$

Remark 2.3. Condition (9) means that we have to choose initial data suiatably, and is a technical restriction. Similarly to Appendix $B$ in [5], we can verify that such $\left(y_{10}, y_{20}\right)$ exists.

The main result of this paper is stated as follows.

Theorem 2.1. There exists a constant $C>0$ such that

$$
\begin{aligned}
& \left|\left(a_{11}-\tilde{a}_{11}, a_{22}-\tilde{a}_{22}\right)\right|_{L^{2}(\Omega)} \\
& \leq C\left(\left.\left.|\ln |\left|y_{1}\left(a_{11}, a_{22}\right)-\tilde{y}_{1}\left(\tilde{a}_{11}, \tilde{a}_{22}\right)\right|\right|_{H^{1}\left(0, T ; H^{1}(\omega)\right)}\right|^{-1}\right. \\
& \left.\quad+|| y_{1}\left(a_{11}, a_{22}\right)-\tilde{y}_{1}\left(\tilde{a}_{11}, \tilde{a}_{22}\right) \|_{H^{1}\left(0, T ; H^{1}(\omega)\right)}\right),
\end{aligned}
$$

for all $\left(a_{11}, a_{22}\right),\left(\tilde{a}_{11}, \tilde{a}_{22}\right) \in \mathcal{A}(\tilde{\omega}, M)$. 
Remark 2.4. One can consider the problem that all the coefficients $\left\{a_{j k}\right\}_{j, k=1}^{2}$ are unknown. In this case, the following three conditions are needed: (1) the unknown coefficient $a_{21}$ must be nonzero in a nonempty open subset $\omega$; (2) the functions $a_{11}$ and $a_{12}, a_{21}$ and $a_{22}$ must be linearity independence, respectively; (3) two times of observations with different suitable chosen initial data of $y_{1}$ are required. As pointed in Remark 2.1, condition (1) can not be removed since we only observe a single component of the solutions. Condition (2) is reasonable since what we can observe is only the linear combination of the coefficients. Condition (3) can not be deleted because for each observation we only observe the linear combinations to get the coefficients from these combinations and we need observe the system twice.

Remark 2.5. From the proof of Theorem 2.1, one can see that it can be generalized to a system coupled by more than two Schrödinger equations with an observation on some components of the solution. In this paper, to present the key idea in a simple way, we do not pursue the full technical generality.

\section{Proof of Theorem 2.1}

Before giving the proof, we present a preliminary result.

Lemma 3.1. For all $\left(a_{11}, a_{22}\right),\left(\tilde{a}_{11}, \tilde{a}_{22}\right) \in \mathcal{A}(\tilde{\omega}, M)$,

$$
\sum_{j=1}^{2}\left\|a_{j j}-\tilde{a}_{j j}\right\|_{L^{2}(\Omega)}^{2} \leq C \sum_{j=1}^{2}\left\|y_{j}\left(a_{11}, a_{22}\right)-\tilde{y}_{j}\left(\tilde{a}_{11}, \tilde{a}_{22}\right)\right\|_{H^{1}\left(0, T ; H^{1}\left(\omega_{1}\right)\right)}^{2} .
$$

In order to obtain the Lipschitz stabilty in (11), the subdomain $\omega_{1}$ can not be arbitrarily small and must satisfy (4). Lemma 3.1 should be a known result. However, since we failed to find an exact reference, we provide it here for the sake of completeness and readers' convenience.

Proof of Lemma 3.1. Let $\phi \in C^{4}(\bar{\Omega})$ be the function satisfying (3) and (4). Set

$$
\hat{\varphi}(x, t) \triangleq \frac{e^{\eta \phi(x)}}{(T+t)(T-t)}, \quad \hat{\alpha}(x, t) \triangleq \frac{e^{2 \eta\|\phi\|_{L} \infty(\Omega)}-e^{\eta \phi(x)}}{(T+t)(T-t)}, \quad \forall(x, t) \in \Omega \times(0, T),
$$

where $\eta$ denotes some positive number which can be specified later.

For $j=1,2$, let

$$
z_{j}=y_{j}\left(a_{11}, a_{22}\right)-\tilde{y}_{j}\left(\tilde{a}_{11}, \tilde{a}_{22}\right), \quad f_{j}(x)=a_{j j}(x)-\tilde{a}_{j j}(x), \quad R_{j}(x, t)=\tilde{y}_{j}(x, t) .
$$


Then $\left(z_{1}, z_{2}\right) \in\left[C\left([0, T] ; H_{0}^{1}(\Omega)\right)\right]^{2}$ is the solution of the following system:

$$
\begin{cases}i \partial_{t} z_{1}+\Delta z_{1}+a_{11} z_{1}+a_{12} z_{2}=f_{1}(x) R_{1}(x, t) & \text { in } \Omega \times(0, T), \\ i \partial_{t} z_{2}+\Delta z_{2}+a_{21} z_{1}+a_{22} z_{2}=f_{2}(x) R_{2}(x, t) & \text { in } \Omega \times(0, T), \\ z_{1}(x, 0)=z_{2}(x, 0)=0 & \text { in } \Omega, \\ z_{1}=z_{2}=0 & \text { on } \Gamma \times(0, T) .\end{cases}
$$

Take the even-conjugate extensions of $\left(z_{1}, z_{2}\right)$ to the interval $(-T, T)$, i.e., set

$$
\left(z_{1}(x, t), z_{2}(x, t)\right)=\left(\overline{z_{1}(x,-t)}, \overline{z_{2}(x,-t)}\right) \quad \text { for } t \in(-T, 0)
$$

If $\left(R_{1}(x, 0), R_{2}(x, 0)\right) \in \mathbb{R}^{2}$ for a.e. $x \in \Omega$, then we set

$$
\left(R_{1}(x, t), R_{2}(x, t)\right)=\left(\overline{R_{1}(x,-t)}, \overline{R_{2}(x,-t)}\right) \quad \text { for } t \in(-T, 0) .
$$

If $\left(i R_{1}(x, 0), i R_{2}(x, 0)\right) \in \mathbb{R}^{2}$ for a.e. $x \in \Omega$, then we set

$$
\left(R_{1}(x, t), R_{2}(x, t)\right)=\left(-\overline{R_{1}(x,-t)},-\overline{R_{2}(x,-t)}\right) \quad \text { for } t \in(-T, 0) .
$$

In such context, we have that $\left(R_{1}, R_{2}\right) \in H^{1}\left(-T, T ; L^{\infty}(\Omega)\right)^{2}$, and $\left(z_{1}, z_{2}\right)$ solves the system (13) in $\Omega \times(-T, T)$.

Assume $\left(u_{1}, u_{2}\right)=\left(\partial_{t} z_{1}, \partial_{t} z_{2}\right)$. We have

$$
\begin{cases}i \partial_{t} u_{1}+\Delta u_{1}+a_{11} u_{1}+a_{12} u_{2}=f_{1}(x) \partial_{t} R_{1}(x, t) & \text { in } \Omega \times(0, T), \\ i \partial_{t} u_{2}+\Delta u_{2}+a_{21} u_{1}+a_{22} u_{2}=f_{2}(x) \partial_{t} R_{2}(x, t) & \text { in } \Omega \times(0, T), \\ u_{1}(x, 0)=-i f_{1}(x) R_{1}(x, 0), \quad u_{2}(x, 0)=-i f_{2}(x) R_{2}(x, 0) & \text { in } \Omega, \\ u_{1}=u_{2}=0 & \text { on } \Gamma \times(0, T) .\end{cases}
$$

It follows from (6), (13) and (14) that $\left(u_{1}, u_{2}\right) \in\left[C^{1}\left([-T, T] ; H_{0}^{1}(\Omega)\right) \cap C([-T, T]\right.$; $\left.\left.H^{2}(\Omega)\right)\right]^{2}$. Further, there exists a constant $\mathbf{C}=\mathbf{C}(M, T)>0$ such that

$$
\left\|\left(u_{1}, u_{2}\right)\right\|_{\left[L^{2}\left(-T, T ; H^{2}(\Omega)\right)\right]^{2}}^{2}+\left\|\left(\partial_{t} u_{1}, \partial_{t} u_{2}\right)\right\|_{\left[L^{2}\left(-T, T ; H_{0}^{1}(\Omega)\right)\right]^{2}}^{2} \leq \mathbf{C} .
$$

For $j=1,2$ and $\tau>0$, let $\hat{u}_{j}=e^{-\tau \hat{\alpha}} u_{j}$ and

$$
\left\{\begin{array}{l}
M_{j 1} \triangleq i\left(2 \tau \nabla \hat{\alpha} \cdot \nabla \hat{u}_{j}+\tau \Delta \hat{\alpha} \hat{u}_{j}\right)+\tau \partial_{t} \hat{\alpha} \hat{u}_{j} \\
M_{j 2} \triangleq \partial_{t} \hat{u}_{j}+i\left(\Delta \hat{u}_{j}+\tau^{2}|\nabla \hat{\alpha}|^{2} \hat{u}_{j}\right)
\end{array}\right.
$$


By Proposition 3.1 in [17], we know that there exist $\tau_{0}>0$ and $\eta_{0}\left(\tau_{0}\right)>0$ such that for all $\tau \geq \tau_{0}$ and $\eta \geq \eta_{0}\left(s_{0}\right)$, it holds that

$$
\begin{aligned}
& \int_{-T}^{T} \int_{\Omega} e^{-2 \tau \hat{\alpha}} \tau^{3} \eta^{4} \hat{\varphi}^{3}\left(\left|u_{1}\right|^{2}+\left|u_{2}\right|^{2}\right) d x d t+\int_{-T}^{T} \int_{\Omega} \sum_{j=1}^{2}\left|M_{j 2}\right|^{2} d x d t \\
& \leq C\left\{\int_{-T}^{T} \int_{\omega_{1}} e^{-2 \tau \hat{\alpha}}\left[\tau^{3} \eta^{4} \hat{\varphi}^{3}\left(\left|u_{1}\right|^{2}+\left|u_{2}\right|^{2}\right)+\tau \eta^{2} \hat{\varphi}\left(\left|\nabla u_{1}\right|^{2}+\left|\nabla u_{2}\right|^{2}\right)\right] d x d t\right. \\
& \left.\quad+\int_{-T}^{T} \int_{\Omega} e^{-2 \tau \hat{\alpha}}\left(\left|f_{1}(x) \partial_{t} R_{1}(x, t)\right|^{2}+\left|f_{2}(x) \partial_{t} R_{2}(x, t)\right|^{2}\right) d x d t\right\}
\end{aligned}
$$

Put

$$
J=-\int_{0}^{T} \int_{\Omega} e^{-\tau \hat{\alpha}} M_{12} \bar{u}_{1} d x d t-\int_{0}^{T} \int_{\Omega} e^{-\tau \hat{\alpha}} M_{22} \bar{u}_{2} d x d t
$$

Then

$$
\begin{aligned}
\operatorname{Re}(J)= & -\operatorname{Re}\left[\int_{0}^{T} \int_{\Omega} \partial_{t} \hat{u}_{1} \overline{\hat{u}}_{1} d x d t+i \int_{0}^{T} \int_{\Omega}\left(-\left|\nabla \hat{u}_{1}\right|^{2}+\tau^{2}|\nabla \hat{\alpha}|^{2}\left|\hat{u}_{1}\right|^{2}\right) d x d t\right] \\
& -\operatorname{Re}\left[\int_{0}^{T} \int_{\Omega} \partial_{t} \hat{u}_{2} \overline{\hat{u}}_{2} d x d t+i \int_{0}^{T} \int_{\Omega}\left(-\left|\nabla \hat{u}_{2}\right|^{2}+\tau^{2}|\nabla \hat{\alpha}|^{2}\left|\hat{u}_{2}\right|^{2}\right) d x d t\right] \\
= & \frac{1}{2} \int_{\Omega}\left(\left|\hat{u}_{1}(x, 0)\right|^{2}+\left|\hat{u}_{2}(x, 0)\right|^{2}\right) d x \\
= & \frac{1}{2} \int_{\Omega} e^{-2 \tau \hat{\alpha}(x, 0)}\left(\left|f_{1}(x)\right|^{2}\left|R_{1}(x, 0)\right|^{2}+\left|f_{2}(x)\right|^{2}\left|R_{2}(x, 0)\right|^{2}\right) d x d t .
\end{aligned}
$$

This, together with the conditions on $R_{1}(x, 0)$ and $R_{2}(x, 0)$, implies that

$$
\operatorname{Re}(J) \geq \frac{r^{2}}{2} \int_{\Omega} e^{-2 \tau \hat{\alpha}(x, 0)}\left(\left|f_{1}(x)\right|^{2}+\left|f_{2}(x)\right|^{2}\right) d x d t .
$$

On the other hand, it follows from (18) that

$$
\begin{aligned}
|J| \leq & \left(\int_{0}^{T} \int_{\Omega} e^{-2 \tau \hat{\alpha}}\left|u_{1}\right|^{2} d x d t\right)^{\frac{1}{2}}\left(\int_{0}^{T} \int_{\Omega}\left|M_{12}\right|^{2} d x d t\right)^{\frac{1}{2}} \\
& +\left(\int_{0}^{T} \int_{\Omega} e^{-2 \tau \hat{\alpha}}\left|u_{2}\right|^{2} d x d t\right)^{\frac{1}{2}}\left(\int_{0}^{T} \int_{\Omega}\left|M_{22}\right|^{2} d x d t\right)^{\frac{1}{2}} \\
\leq & \tau^{\frac{3}{2}} \eta^{2} \int_{0}^{T} \int_{\Omega} e^{-2 \tau \hat{\alpha}}\left(\left|u_{1}\right|^{2}+\left|u_{2}\right|^{2}\right) d x d t+\tau^{-\frac{3}{2}} \eta^{-2} \int_{0}^{T} \int_{\Omega}\left(\left|M_{12}\right|^{2}+\left|M_{22}\right|^{2}\right) d x d t .
\end{aligned}
$$


From the choice of $\hat{\alpha}$, we find that

$$
\begin{aligned}
& \int_{-T}^{T} \int_{\Omega} e^{-2 \tau \hat{\alpha}}\left(\left|f_{1}(x) \partial_{t} R_{1}(x, t)\right|^{2}+\left|f_{2}(x) \partial_{t} R_{2}(x, t)\right|^{2}\right) d x d t \\
\leq & C \int_{\Omega} e^{-2 \tau \hat{\alpha}(x, 0)}\left(\left|f_{1}(x)\right|^{2}+\left|f_{2}(x)\right|^{2}\right) d x .
\end{aligned}
$$

This, together with (17), (19) and (20), implies that

$$
\begin{gathered}
\frac{r^{2}}{2} \int_{\Omega} e^{-2 \tau \hat{\alpha}(x, 0)}\left(\left|f_{1}(x)\right|^{2}+\left|f_{2}(x)\right|^{2}\right) d x d t \\
\leq C \tau^{-\frac{3}{2}} \eta^{-2}\left\{\int_{-T}^{T} \int_{\omega_{1}} e^{-2 \tau \hat{\alpha}}\left[\tau^{3} \eta^{4} \hat{\varphi}^{3}\left(\left|u_{1}\right|^{2}+\left|u_{2}\right|^{2}\right)+\tau \eta^{2} \hat{\varphi}\left(\left|\nabla u_{1}\right|^{2}+\left|\nabla u_{2}\right|^{2}\right)\right] d x d t\right. \\
\left.\quad+\int_{\Omega} e^{-2 \tau \hat{\alpha}(x, 0)}\left(\left|f_{1}(x)\right|^{2}+\left|f_{2}(x)\right|^{2}\right) d x\right\} .
\end{gathered}
$$

Thus, there is an $\tau_{1}>0$ such that for all $\tau \geq \max \left\{\tau_{0}, \tau_{1}\right\}$ and $\eta \geq \eta_{0}\left(\tau_{0}\right)$,

$$
\begin{aligned}
& \int_{\Omega} e^{-2 \tau \hat{\alpha}(x, 0)}\left(\left|f_{1}(x)\right|^{2}+\left|f_{2}(x)\right|^{2}\right) d x d t \\
\leq & C \tau^{-\frac{3}{2}} \eta^{-2} \int_{-T}^{T} \int_{\omega_{1}} e^{-2 \tau \hat{\alpha}}\left[\tau^{3} \eta^{4} \hat{\varphi}^{3}\left(\left|u_{1}\right|^{2}+\left|u_{2}\right|^{2}\right)+\tau \eta^{2} \hat{\varphi}\left(\left|\nabla u_{1}\right|^{2}+\left|\nabla u_{2}\right|^{2}\right)\right] d x d t .
\end{aligned}
$$

This concludes (11) and completes the proof of Lemma 3.1.

Next, in order to keep the self-containment, we give a brief introduction to F.B.I. transformation here. Let

$$
F(z)=\frac{1}{2 \pi} \int_{\mathbb{R}} e^{i z \varrho} e^{-\varrho^{2}} d \varrho .
$$

Then

$$
F(z)=\frac{\sqrt{\pi}}{2 \pi} e^{\frac{1}{4}\left(|\operatorname{Im} z|^{2}-|\operatorname{Re} z|^{2}\right)} e^{-\frac{i}{2}(\operatorname{Im} z \operatorname{Re} z)} .
$$

For every $\lambda \geq 1$, define

$$
F_{\lambda}(z) \triangleq \lambda F(\lambda z)=\frac{1}{2 \pi} \int_{\mathbb{R}} e^{i z \varrho} e^{-\left(\frac{\rho}{\lambda}\right)^{2}} d \tau
$$

Then,

$$
\left|F_{\lambda}(z)\right|=\frac{\sqrt{\pi}}{2 \pi} \lambda e^{\frac{\lambda^{2}}{4}\left(|\operatorname{Im} z|^{2}-|\operatorname{Re} z|^{2}\right)} .
$$

Let $s, l_{0} \in \mathbb{R}$, the F.B.I. transformation $\mathcal{F}_{\lambda}$ for $u \in \mathcal{S}\left(\mathbb{R}^{n+1}\right)$ is defined as follows:

$$
\left(\mathcal{F}_{\lambda} u\right)(x, s)=\int_{\mathbb{R}} F_{\lambda}\left(l_{0}+i s-l\right) \Phi(l) u(x, l) d l .
$$

Now we are in a position to prove Theorem 2.1. 
Proof of Theorem 2.1. The proof is long. We divide it into four steps.

Step 1. In this step, we introduce an equation on $(-T, T) \times \tilde{\omega}$.

Recall that $\omega$ is an arbitrary fixed nonempty subset of $\tilde{\omega}$ such that $\bar{\omega} \subset \tilde{\omega}$. By $[11$, Lemma 1.1], there exists a function $\psi \in C^{2}(\overline{\tilde{\omega}})$ such that

$$
\left\{\begin{array}{l}
\psi(x)>0, \quad \forall x \in \tilde{\omega} \\
\psi(x)=0, \quad \forall x \in \partial \tilde{\omega} \\
|\nabla \psi(x)|>0, \quad \forall x \in \overline{\tilde{\omega} \backslash \omega} .
\end{array}\right.
$$

We can conclude from (24) that there exist a constant $\beta>0$ and $\omega_{2} \subset \subset \tilde{\omega}$ such that

$$
\psi(x) \leq \beta, \quad \forall x \in \tilde{\omega} \backslash \omega_{2}
$$

and that

$$
\psi(x) \geq 2 \beta, \quad \forall x \in \omega_{1} .
$$

It follows from the last condition in (24) that the maximum value of $\psi$ can only be attained in $\omega$, i.e., there exists a point $x_{0} \in \omega$ such that

$$
\psi\left(x_{0}\right)=\max _{x \in \tilde{\omega}} \psi(x) .
$$

Let $\chi \in C_{0}^{\infty}(\tilde{\omega})$ be a cut-off function, which satisfies $0 \leq \chi \leq 1$ and

$$
\chi(x)= \begin{cases}1, & \text { if } x \in \omega_{2}, \\ 0, & \text { if } x \in \tilde{\omega} \backslash \omega_{3},\end{cases}
$$

where $\omega_{3}$ is a subset of $\tilde{\omega}$ such that $\omega_{2} \subset \subset \omega_{3}$.

Let $\left(w_{1}, w_{2}\right)=\left(\chi u_{1}, \chi u_{2}\right)$. Then by $(8)$ and (14), we have that

$$
\begin{cases}i \partial_{t} w_{1}+\Delta w_{1}+a_{11} w_{1}+a_{12} w_{2}=[\Delta, \chi] u_{1} & \text { in } \tilde{\omega} \times(0, T), \\ i \partial_{t} w_{2}+\Delta w_{2}+a_{21} w_{1}+a_{22} w_{2}=[\Delta, \chi] u_{2} & \text { in } \tilde{\omega} \times(0, T), \\ w_{1}(0)=w_{2}(0)=0 & \text { in } \tilde{\omega}, \\ w_{1}=w_{2}=0 & \text { on } \partial \tilde{\omega} \times(0, T) .\end{cases}
$$

By (15), there exists $\mathbf{C}=\mathbf{C}(M, T)>0$ such that

$$
\left\|\left(w_{1}, w_{2}\right)\right\|_{L^{2}\left(-T, T ; H^{2}(\widetilde{\omega})\right)^{2}}^{2}+\left\|\left(\partial_{t} w_{1}, \partial_{t} w_{2}\right)\right\|_{L^{2}\left(-T, T ; H_{0}^{1}(\widetilde{\omega})\right)^{2}}^{2} \leq \mathbf{C} .
$$

Step 2. In this step, we introduce a system of parabolic equations related to (29) and a Carleman estimate to the parabolic system. 
For $j=1,2$, let $W_{j}(x, s)=\int_{\mathbb{R}} F_{\lambda}\left(l_{0}+i s-l\right) \Phi(l) w_{j}(x, l) d l$. Since

$$
\begin{aligned}
\partial_{s} W_{j}(x, s) & =\int_{\mathbb{R}}-i \partial_{l} F_{\lambda}\left(l_{0}+i s-l\right) \Phi(l) w_{j}(x, l) d l \\
& =i \int_{\mathbb{R}} F_{\lambda}\left(l_{0}+i s-l\right)\left(\Phi^{\prime}(l) w(x, l)+\Phi(l) w_{t}(x, l)\right) d l,
\end{aligned}
$$

we follow that

$$
\begin{cases}\partial_{s} W_{1}+\Delta W_{1}+a_{11} W_{1}+a_{12} W_{2}=F_{1}+G_{1} & \text { in } \tilde{\omega} \times \mathbb{R}, \\ \partial_{s} W_{2}+\Delta W_{2}+a_{21} W_{1}+a_{22} W_{2}=F_{2}+G_{2} & \text { in } \tilde{\omega} \times \mathbb{R}, \\ W_{1}=W_{2}=0 & \text { on } \partial \tilde{\omega} \times \mathbb{R},\end{cases}
$$

where for $j=1,2$,

$$
\begin{aligned}
& F_{j}(x, s)=i \int_{\mathbb{R}} F_{\lambda}\left(l_{0}+i s-l\right) \Phi^{\prime}(l) w_{j}(x, l) d l \\
& G_{j}(x, s)=\int_{\mathbb{R}} F_{\lambda}\left(l_{0}+i s-l\right) \Phi(l)[\Delta, \chi] u_{j} d l .
\end{aligned}
$$

Let

$$
\varphi(x, t)=\frac{e^{\eta \psi(x)}}{(T+t)(T-t)}, \quad \alpha(x, t)=\frac{e^{\eta \psi(x)}-e^{2 \eta\|\psi\|_{C(\bar{\omega})}}}{(T+t)(T-t)}, \quad \forall(x, t) \in \tilde{\omega} \times(-T, T),
$$

where $\eta>0$.

Let $\Phi \in C_{0}^{\infty}(\mathbb{R})$ satisfying the following conditions:

$$
\left\{\begin{array}{l}
\Phi \in C_{0}^{\infty}\left(\left[-\frac{L}{2}, \frac{L}{2}\right] ;[0,1]\right), \\
\Phi=1 \text { on }\left[-\frac{L}{4}, \frac{L}{4}\right], \\
\left|\Phi^{\prime}\right| \leq \frac{2}{L},
\end{array}\right.
$$

where $L>0$ will be chosen later.

Take

$$
K=\left[-\frac{L}{2},-\frac{L}{4}\right] \bigcup\left[\frac{L}{4}, \frac{L}{2}\right], \quad K_{0}=\left[-\frac{L}{8}, \frac{L}{8}\right] .
$$

Then $l_{0} \in K_{0}$ in $(23)$.

According to Theorem 1.1 in [13], there exist a positive function $\alpha_{0} \in C^{2}(\overline{\tilde{\omega}})$ (only depending on $\tilde{\omega}$ and $\omega$ ), two positive constants $C_{0}$ (only depending on $\tilde{\omega}, \omega, \alpha_{0}$ and $M_{0}$ ) 
and $\sigma_{0}=\sigma_{0}\left(\tilde{\omega}, \omega, M_{0}\right)$ such that the solution $\left(W_{1}, W_{2}\right) \in\left[C\left([-T, T] ; L^{2}(\tilde{\omega})\right) \cap L^{2}([-T, T]\right.$; $\left.\left.H^{1}(\tilde{\omega})\right)\right]^{2}$ of $(31)$ satisfies that

$$
\begin{aligned}
& \int_{-T}^{T} \int_{\tilde{\omega}}\left(\sigma^{4} \gamma(s)^{4}\left|\nabla W_{1}\right|^{2}+\sigma \gamma(s)\left|\nabla W_{2}\right|^{2}+\sigma^{6} \gamma(s)^{6}\left|W_{1}\right|^{2}+\sigma^{3} \gamma(s)^{3}\left|W_{2}\right|^{2}\right) e^{2 \sigma \alpha} d x d s \\
& \leq C_{0}\left[\int_{-T}^{T} \int_{\tilde{\omega}}\left(\sigma^{3} \gamma(s)^{3}\left|F_{1}(x, s)+G_{1}(x, s)\right|^{2}+\left|F_{2}(x, s)+G_{2}(x, s)\right|^{2}\right) e^{2 \sigma \alpha} d x d s\right. \\
& \left.\quad+\sigma^{7} \int_{-T}^{T} \int_{\omega} e^{2 \sigma \alpha} \gamma(s)^{7}\left|W_{1}\right|^{2} d x d s\right],
\end{aligned}
$$

where $\gamma(s)=\frac{1}{(T+s)(T-s)}$ and $\sigma \geq \sigma_{0}$.

Step 3. In this step, we estimate all the terms in the right hand side of (33).

Let

$$
\mu_{2}=\frac{e^{2 \eta \psi\left(x_{0}\right)}-e^{\eta \psi\left(x_{0}\right)}}{T^{2}}
$$

There exists $\delta_{2}>0$ such that

$$
\max _{x \in \omega, t \in[-T, T]} \gamma(s)^{7} e^{2 \sigma \alpha} \leq e^{-\left(2-\delta_{2}\right) \sigma \mu_{2}} .
$$

By the property of F.B.I. transformation, we have that

$$
\begin{aligned}
& \int_{-T}^{T} \int_{\omega} \gamma(s)^{7}\left|W_{1}\right|^{2} e^{2 \sigma \alpha} d x d s \\
\leq & \max _{x \in \omega, s \in[-T, T]}\left(\gamma(s)^{7} e^{2 \sigma \alpha}\right) \int_{-T}^{T} \int_{\omega}\left|W_{1}(x, s)\right|^{2} d x d s \\
\leq & e^{-\left(2-\delta_{2}\right) \sigma \mu_{2}} \int_{-T}^{T} \int_{\omega}\left|\int_{\mathbb{R}} F_{\lambda}\left(l_{0}+i s-l\right) \Phi(l) w_{1}(x, l) d l\right|^{2} d x d s \\
\leq & e^{-\left(2-\delta_{2}\right) \sigma \mu_{2}} \int_{-T}^{T} \int_{\omega}\left|\int_{\mathbb{R}} \frac{\sqrt{\pi}}{2 \pi} \lambda e^{\frac{\lambda^{2}}{4}\left(s^{2}-\left|l_{0}-l\right|^{2}\right)} \Phi(l) w_{1}(x, l) d l\right|^{2} d x d s \\
\leq & \frac{\lambda^{2}}{4 \pi} e^{-\left(2-\delta_{2}\right) \sigma \mu_{2}} \int_{-T}^{T} e^{\frac{\lambda^{2}}{2} s^{2}} d s|\sup \Phi|^{2} \int_{\omega}\left|\int_{-\frac{L}{2}}^{\frac{L}{2}} w_{1}(x, l) d l\right|^{2} d x \\
\leq & \frac{\lambda^{2} L T}{2 \pi} e^{\left.-\left(2-\delta_{2}\right)\right) \sigma \mu_{2}} e^{\frac{\lambda^{2}}{2} T^{2}} \int_{\omega} \int_{-\frac{L}{2}}^{\frac{L}{2}}\left|w_{1}(x, l)\right|^{2} d l d x .
\end{aligned}
$$

From the definition of $F_{j}$, we see that

$$
\int_{-T}^{T} \int_{\tilde{\omega}}\left|F_{j}(x, s)\right|^{2} d x d s
$$




$$
\begin{aligned}
& =\int_{-T}^{T} \int_{\tilde{\omega}}\left|i \int_{\mathbb{R}} F_{\lambda}\left(l_{0}+i s-l\right) \Phi^{\prime}(l) w_{j}(x, l) d l\right|^{2} d x d s \\
& \leq \int_{-T}^{T} \int_{\tilde{\omega}}\left|\int_{K} \frac{\sqrt{\pi}}{2 \pi} \lambda e^{\frac{\lambda^{2}}{4}\left(s^{2}-\left|l_{0}-l\right|^{2}\right)} \Phi^{\prime}(l) w_{j}(x, l) d l\right|^{2} d x d s \\
& \leq \frac{1}{2 \pi} \lambda^{2} e^{\frac{\lambda^{2}}{2} T^{2}} T \max _{K}\left|\Phi^{\prime}(l)\right|^{2} \int_{\tilde{\omega}}\left|\int_{K} e^{-\frac{\lambda^{2}}{4}\left|l_{0}-l\right|^{2}} w_{j}(x, l) d l\right|^{2} d x \\
& \leq \frac{1}{2 \pi} \lambda^{2} e^{\frac{\lambda^{2}}{2} T^{2}} T e^{-\frac{\lambda^{2}}{2}\left(\frac{L}{8}\right)^{2}} \max _{K}\left|\Phi^{\prime}(l)\right|^{2} \frac{L}{2} \int_{\tilde{\omega}} \int_{K}\left|w_{j}(x, l)\right|^{2} d l d x \\
& \leq \frac{1}{2 \pi} \lambda^{2} e^{\frac{\lambda^{2}}{2} T^{2}} T e^{-\frac{\lambda^{2}}{2}\left(\frac{L}{8}\right)^{2}}\left(\frac{2}{L}\right)^{2} \frac{L}{2} \int_{\tilde{\omega}} \int_{K}\left|w_{j}(x, l)\right|^{2} d l d x \\
& \leq \frac{\lambda^{2} T}{\pi L} e^{\frac{\lambda^{2}}{2}\left(T^{2}-\left(\frac{L}{8}\right)^{2}\right)} \int_{\tilde{\omega}} \int_{K}\left|w_{j}(x, l)\right|^{2} d l d x .
\end{aligned}
$$

Since $\operatorname{supp} \chi^{\prime} \subset \tilde{\omega} \backslash \omega_{1}$ and $G_{j}(x, s)=0$ in $\omega_{1}$, it holds that

$$
\begin{aligned}
& \int_{-T}^{T} \int_{\tilde{\omega}}\left|G_{j}(x, s)\right|^{2} d x d s \\
= & \int_{-T}^{T} \int_{\tilde{\omega}}\left|\int_{\mathbb{R}} F_{\lambda}\left(l_{0}+i s-l\right) \Phi(l)[\Delta, \chi] u_{j} d l\right|^{2} d x d s \\
\leq & \int_{-T}^{T} \int_{\tilde{\omega}}\left|\int_{-\frac{L}{2}}^{\frac{L}{2}} \frac{\sqrt{\pi}}{2 \pi} \lambda e^{\frac{\lambda^{2}}{4}\left(s^{2}-\left.\left|l_{0}-l\right|\right|^{2}\right)}[\Delta, \chi] u_{j} d l\right|^{2} d x d s \\
\leq & \frac{1}{2 \pi} \lambda^{2} e^{\frac{\lambda^{2}}{2} T^{2}} T \int_{\tilde{\omega} \backslash \omega_{1}}\left|\int_{-\frac{L}{2}}^{\frac{L}{2}} e^{-\frac{\lambda^{2}}{4}\left|l_{0}-l\right|^{2}}[\Delta, \chi] u_{j} d l\right|^{2} d x \\
\leq & \frac{\lambda^{2} T L}{2 \pi} e^{\frac{\lambda^{2}}{2} T^{2}} \max \left\{|\nabla \chi|^{2},|\Delta \chi|^{2}\right\} \int_{-\frac{L}{2}}^{\frac{L}{2}} \int_{\tilde{\omega} \backslash \omega_{1}}\left(\left|u_{j}(x, l)\right|^{2}+\left|\nabla u_{j}(x, l)\right|^{2}\right) d x d l .
\end{aligned}
$$

Set

$$
\mu_{1}=\frac{e^{2 \eta\|\psi \psi\|_{\infty}}-e^{\eta \beta}}{T^{2}} .
$$

By (25), we know that there exists $\delta_{1}>0$ such that

$$
\max _{x \in \tilde{\omega}, s \in[-T, T]} \gamma(s)^{3} e^{2 \sigma \alpha} \leq e^{-\left(2-\delta_{1}\right) \sigma \mu_{2}}, \quad \max _{x \in \tilde{\omega} \backslash \omega_{1}, s \in[-T, T]} \gamma(s)^{3} e^{2 \sigma \alpha} \leq e^{-\left(2-\delta_{1}\right) \sigma \mu_{1}} .
$$

Consequently,

$$
\begin{aligned}
& \int_{-T}^{T} \int_{\tilde{\omega}} e^{2 \sigma \alpha} \gamma(t)^{3}\left|F_{j}(x, s)\right|^{2} d x d s \\
\leq & e^{-\left(2-\delta_{2}\right) \sigma \mu_{2}} \frac{\lambda^{2} T}{\pi L} e^{\frac{\lambda^{2}}{2}\left(T^{2}-\left(\frac{L}{8}\right)^{2}\right)} \int_{\tilde{\omega}} \int_{K}\left|w_{j}(x, l)\right|^{2} d l d x
\end{aligned}
$$


and

$$
\begin{aligned}
& \int_{-T}^{T} \int_{\tilde{\omega}} e^{2 \sigma \alpha} \gamma(t)^{3}\left|G_{j}(x, s)\right|^{2} d x d s \\
\leq & e^{-\left(2-\delta_{1}\right) \sigma \mu_{1}} \frac{\lambda^{2} T L}{2 \pi} e^{\frac{\lambda^{2}}{2} T^{2}} \max \left\{|\nabla \chi|^{2},|\Delta \chi|^{2}\right\} \int_{-\frac{L}{2}}^{\frac{L}{2}} \int_{\tilde{\omega} \backslash \omega_{1}}\left(\left|u_{j}(x, l)\right|^{2}+\left|\nabla u_{j}(x, l)\right|^{2}\right) d x d l .
\end{aligned}
$$

Substituting (35), (39) and (40) into (33), we obtain that

$$
\begin{gathered}
\int_{-T}^{T} \int_{\tilde{\omega}}\left[\sigma^{4} \gamma(s)^{4}\left|\nabla W_{1}\right|^{2}+\sigma \gamma(s)\left|\nabla W_{2}\right|^{2}+\sigma^{6} \gamma(s)^{6}\left|W_{1}\right|^{2}+\sigma^{3} \gamma(s)^{3}\left|W_{2}\right|^{2}\right] e^{2 \sigma \alpha} d x d s \\
\leq C_{0}\left[e^{-\left(2-\delta_{1}\right) \sigma \mu_{2}} \frac{2 \lambda^{2} T}{\pi L} e^{\frac{\lambda^{2}}{2}\left(T^{2}-\left(\frac{L}{8}\right)^{2}\right)} \int_{\tilde{\omega}} \int_{K}\left(\sigma^{3}\left|w_{1}(x, l)\right|^{2}+\left|w_{2}(x, l)\right|^{2}\right) d l d x\right. \\
+e^{-\left(2-\delta_{1}\right) \sigma \mu_{1}} \frac{\lambda^{2} T L}{\pi} e^{\frac{\lambda^{2}}{2} T^{2}} \max \left\{|\nabla \chi|^{2},|\Delta \chi|^{2}\right\} \int_{-\frac{L}{2}}^{\frac{L}{2}} \int_{\tilde{\omega} \backslash \omega_{1}}\left[\sigma^{3}\left(\left|u_{1}(x, l)\right|^{2}+\left|\nabla u_{1}(x, l)\right|^{2}\right)\right. \\
\left.+\left(\left|u_{2}(x, l)\right|^{2}+\left|\nabla u_{2}(x, l)\right|^{2}\right)\right] d x d l \\
\left.+\sigma^{7} \frac{\lambda^{2} L T}{2 \pi} e^{-\left(2-\delta_{2}\right) \sigma \mu_{2}} e^{\frac{\lambda^{2}}{2} T^{2}} \int_{-\frac{L}{2}}^{\frac{L}{2}} \int_{\omega}\left(\left|w_{1}(x, l)\right|^{2}+\left|\nabla w_{1}(x, l)\right|^{2}\right) d x d l\right] .
\end{gathered}
$$

In order to reduce the computation complexity of the proof, and without loss of generality, in the following steps, we assume $T=1$. Let $A>1$. By choosing $L=8 A T=8 A$, we have

$$
\begin{aligned}
& e^{-2 \sigma \mu_{3}} \sigma^{4} \int_{-1+\epsilon}^{1-\epsilon} \int_{\omega_{1}}\left(\left|\nabla W_{1}\right|^{2}+\left|W_{1}\right|^{2}\right) d x d s \\
& \leq \int_{-1}^{1} \int_{\tilde{\omega}}\left[\sigma^{4} \gamma(s)^{4}\left|\nabla W_{1}\right|^{2}+\sigma^{6} \gamma(s)^{6}\left|W_{1}\right|^{2}\right] e^{-2 \sigma \alpha} d x d s \\
& \leq C_{0}\left[e^{-\left(2-\delta_{1}\right) \sigma \mu_{2}} \frac{2 \lambda^{2}}{8 \pi A} e^{\frac{\lambda^{2}}{2}\left(1-A^{2}\right)} \int_{\tilde{\omega}} \int_{K}\left[\sigma^{3}\left|w_{1}(x, l)\right|^{2}+\left|w_{2}(x, l)\right|^{2}\right] d l d x\right. \\
& \quad+e^{-\left(2-\delta_{1}\right) \sigma \mu_{1}} \frac{8 A \lambda^{2}}{\pi} e^{\frac{\lambda^{2}}{2}} \max \left\{|\nabla \chi|^{2},|\Delta \chi|^{2}\right\} \int_{-4 A}^{4 A} \int_{\tilde{\omega} \backslash \omega_{1}}\left[\sigma^{3}\left(\left|u_{1}(x, l)\right|^{2}+\left|\nabla u_{1}(x, l)\right|^{2}\right)\right. \\
& \left.\quad+\left(\left|u_{2}(x, l)\right|^{2}+\left|\nabla u_{2}(x, l)\right|^{2}\right)\right] d x d l \\
& \left.\quad+\sigma^{7} \frac{4 A \lambda^{2}}{\pi} e^{-\left(2-\delta_{2}\right) \sigma \mu_{2}} e^{\frac{\lambda^{2}}{2}} \int_{-4 A}^{4 A} \int_{\omega}\left(\left|w_{1}(x, l)\right|^{2}+\left|\nabla w_{1}(x, l)\right|^{2}\right) d x d l\right],
\end{aligned}
$$

where

$$
\mu_{3}=\frac{e^{2 \eta\|\psi\|_{\infty}}-e^{2 \eta \beta}}{\epsilon(2-\epsilon)}
$$


Similarly, we can get that

$$
\begin{aligned}
& e^{-2 \sigma \mu_{3}} \sigma \int_{-1+\epsilon}^{1-\epsilon} \int_{\omega_{1}}\left(\left|\nabla W_{2}\right|^{2}+\left|W_{2}\right|^{2}\right) d x d s \\
& \leq \int_{-1}^{1} \int_{\tilde{\omega}}\left[\sigma \gamma(s)\left|\nabla W_{2}\right|^{2}+\sigma^{3} \gamma(s)^{3}\left|W_{2}\right|^{2}\right] e^{-2 \sigma \alpha} d x d s \\
& \leq C_{0}\left[e^{-\left(2-\delta_{1}\right) \sigma \mu_{2}} \frac{2 \lambda^{2}}{8 \pi A} e^{\frac{\lambda^{2}}{2}\left(1-A^{2}\right)} \int_{\tilde{\omega}} \int_{K}\left[\sigma^{3}\left|w_{1}(x, l)\right|^{2}+\left|w_{2}(x, l)\right|^{2}\right] d l d x\right. \\
& \quad+e^{-\left(2-\delta_{1}\right) \sigma \mu_{1}} \frac{8 A \lambda^{2}}{\pi} e^{\frac{\lambda^{2}}{2}} \max \left\{|\nabla \chi|^{2},|\Delta \chi|^{2}\right\} \int_{-4 A}^{4 A} \int_{\tilde{\omega} \backslash \omega_{1}}\left[\sigma^{3}\left(\left|u_{1}(x, l)\right|^{2}+\left|\nabla u_{1}(x, l)\right|^{2}\right)\right. \\
& \left.\quad+\left(\left|u_{2}(x, l)\right|^{2}+\left|\nabla u_{2}(x, l)\right|^{2}\right)\right] d x d l \\
& \left.\quad+\sigma^{7} \frac{4 A \lambda^{2}}{\pi} e^{-\left(2-\delta_{2}\right) \sigma \mu_{2}} e^{\frac{\lambda^{2}}{2}} \int_{-4 A}^{4 A} \int_{\omega}\left(\left|w_{1}(x, l)\right|^{2}+\left|\nabla w_{1}(x, l)\right|^{2}\right) d x d l\right] .
\end{aligned}
$$

Fix $\epsilon \in(0,1)$ such that

$$
\tau \triangleq \epsilon(2-\epsilon) \frac{2-\delta_{1}}{2} \frac{e^{2 \eta\|\psi\|_{\infty}}-e^{\eta \beta}}{e^{2 \eta\|\psi\|_{\infty}}-e^{2 \eta \beta}}-1>0 .
$$

This is equivalent to say that

$$
\left(2-\delta_{1}\right) \mu_{1}-2 \mu_{3}=2 \tau \mu_{3}>0 .
$$

Hence,

$$
\begin{aligned}
& \int_{-1+\epsilon}^{1-\epsilon} \int_{\omega_{1}}\left(\left|\nabla W_{1}\right|^{2}+\left|W_{1}\right|^{2}\right) d x d t+\frac{1}{\sigma^{3}} \int_{-1+\epsilon}^{1-\epsilon} \int_{\omega_{1}}\left(\left|\nabla W_{2}\right|^{2}+\left|W_{2}\right|^{2}\right) d x d t \\
\leq C_{0} & \frac{1}{\sigma^{4}}\left[\sigma^{3} e^{-\left(2-\delta_{1}\right) \sigma \mu_{2}+2 \sigma \mu_{3}} \frac{\lambda^{2} T}{4 \pi A} e^{\frac{\lambda^{2}}{2}\left(1-A^{2}\right)} \int_{\tilde{\omega}} \int_{K}\left[\left|w_{1}(x, l)\right|^{2}+\left|w_{2}(x, l)\right|^{2}\right] d l d x\right. \\
& +\sigma^{3} \frac{8 A \lambda^{2}}{\pi} e^{-2 \sigma \tau \mu_{3}} e^{\frac{\lambda^{2}}{2}} \max \left\{|\nabla \chi|^{2},|\Delta \chi|^{2}\right\}\left(|| u_{1} \|_{L^{2}\left(-4 A, 4 A ; H^{1}\left(\tilde{\omega} \backslash \omega_{1}\right)\right)}^{2}+\right. \\
& \left.\left.+\left\|u_{2}\right\|_{L^{2}\left(-4 A, 4 A ; H^{1}\left(\tilde{\omega} \backslash \omega_{1}\right)\right)}^{2}\right)+\sigma^{7} \frac{4 \lambda^{2} A}{\pi} e^{\sigma\left(2 \mu_{3}-\left(2-\delta_{2}\right) \mu_{2}\right)} e^{\frac{\lambda^{2}}{2}}\left\|w_{1}\right\|_{L^{2}\left(-4 A, 4 A ; H^{1}(\omega)\right)}^{2}\right] \\
\leq C_{0} & {\left[\frac{\lambda^{2}}{4 \pi \sigma A} e^{-\left(2-\delta_{1}\right) \sigma \mu_{2}+2 \sigma \mu_{3}} e^{\frac{\lambda^{2}}{2}\left(1-A^{2}\right)}\left(\left\|w_{1}\right\|_{L^{2}(K \times \tilde{\omega})}^{2}+\left\|w_{2}\right\|_{L^{2}(K \times \tilde{\omega})}^{2}\right)\right.} \\
& +\frac{8 \lambda^{2} A}{\pi \sigma} e^{-2 \sigma \tau \mu_{3}} e^{\frac{\lambda^{2}}{2}} \max \left\{|\nabla \chi|^{2},|\Delta \chi|^{2}\right\}\left(\left\|u_{1}\right\|_{L^{2}\left(-4 A, 4 A ; H^{1}\left(\tilde{\omega} \backslash \omega_{1}\right)\right)}^{2}\right. \\
& \left.\left.+\left\|u_{2}\right\|_{L^{2}\left(-4 A, 4 A ; H^{1}\left(\tilde{\omega} \backslash \omega_{1}\right)\right)}^{2}\right)+\frac{4 \lambda^{2} \sigma^{3} A}{\pi} e^{\sigma\left(2 \mu_{3}-\left(2-\delta_{2}\right) \mu_{2}\right)} e^{\frac{\lambda^{2}}{2}}\left\|w_{1}\right\|_{L^{2}\left(-4 A, 4 A ; H^{1}(\omega)\right)}^{2}\right] \\
\leq C_{0} & \frac{8 \lambda^{2} A}{\pi \sigma}\left[\frac{1}{32 A^{2}} e^{-\left(2-\delta_{1}\right) \sigma \mu_{2}+2 \sigma \mu_{3}} e^{\frac{\lambda^{2}}{2}\left(1-A^{2}\right)}\left(\left\|w_{1}\right\|_{L^{2}(K \times \tilde{\omega})}^{2}+\left\|w_{2}\right\|_{L^{2}(K \times \tilde{\omega})}^{2}\right)\right.
\end{aligned}
$$




$$
\begin{aligned}
& +e^{-2 \sigma \tau \mu_{3}} e^{\frac{\lambda^{2}}{2}} \max \left\{|\nabla \chi|^{2},|\Delta \chi|^{2}\right\}\left(\left\|u_{1}\right\|_{L^{2}\left(-4 A, 4 A ; H^{1}\left(\tilde{\omega} \backslash \omega_{1}\right)\right)}^{2}\right. \\
& \left.+|| u_{2} \|_{L^{2}\left(-4 A, 4 A ; H^{1}\left(\tilde{\omega} \backslash \omega_{1}\right)\right)}^{2}\right) \\
& \left.+\frac{\sigma^{4}}{2} e^{\sigma\left(2 \mu_{3}-\left(2-\delta_{2}\right) \mu_{2}\right)} e^{\frac{\lambda^{2}}{2} T^{2}}\left\|w_{1}\right\|_{L^{2}\left(-4 A, 4 A ; H^{1}(\omega)\right)}^{2}\right] \\
& \leq C_{0} \frac{16 \lambda^{2} A}{\pi \sigma}\left[\left(e^{-\left(2-\delta_{1}\right) \sigma \mu_{2}+2 \sigma \mu_{3}} \frac{1}{32 A^{2}} e^{\frac{\lambda^{2}}{2}\left(1-A^{2}\right)}+e^{-2 \sigma \tau \mu_{3}} e^{\frac{\lambda^{2}}{2}} \max \left\{|\nabla \chi|^{2},|\Delta \chi|^{2}\right\}\right) \mathbf{C}\right. \\
& \left.+\frac{\sigma^{4}}{2} e^{\sigma\left(2 \mu_{3}-\left(2-\delta_{2}\right) \mu_{2}\right)} e^{\frac{\lambda^{2}}{2}}\left\|w_{1}\right\|_{L^{2}\left(-4 A, 4 A ; H^{1}(\omega)\right)}^{2}\right] .
\end{aligned}
$$

Step 4. By Lemma 3.1,

$$
\begin{aligned}
& \sum_{j=1}^{2}\left\|a_{j j}-\tilde{a}_{j j}\right\|_{L^{2}(\Omega)} \\
\leq & C\left\|\left(y_{1}\left(a_{11}, a_{22}\right)-\tilde{y}_{1}\left(\tilde{a}_{11}, \tilde{a}_{22}\right), y_{2}\left(a_{11}, a_{22}\right)-\tilde{y}_{2}\left(\tilde{a}_{11}, \tilde{a}_{22}\right)\right)\right\|_{\left[H^{1}\left(0, T ; H^{1}(\omega)\right)\right]^{2}} \\
\leq & C\left(\left\|\left(\nabla w_{1}, \nabla w_{2}\right)\right\|_{L^{2}\left(\omega_{1} \times(-T, T)\right)}^{2}+\left\|\left(w_{1}, w_{2}\right)\right\|_{L^{2}\left(\omega_{1} \times(-T, T)\right)}^{2}\right) \\
\leq & C\left(\left\|\left(\nabla\left(\Phi w_{1}\right), \nabla\left(\Phi w_{2}\right)\right)\right\|_{L^{2}\left(\omega_{1} \times\left(-\frac{L}{2}, \frac{L}{2}\right)\right)}^{2}+\left\|\left(\Phi w_{1}, \Phi w_{2}\right)\right\|_{L^{2}\left(\omega_{1} \times\left(-\frac{L}{2}, \frac{L}{2}\right)\right)}^{2}\right) .
\end{aligned}
$$

It follows from Parseval's identity that

$$
\begin{aligned}
& \left.|| \Phi w_{j}\right|_{L^{2}\left(\omega_{1} \times\left(-\frac{L}{2}, \frac{L}{2}\right)\right)} ^{2}=\int_{-\frac{L}{2}}^{\frac{L}{2}} \int_{\omega_{1}}\left|\Phi(t) w_{j}(x, t)\right|^{2} d x d t \\
= & \int_{\mathbb{R}} \int_{\omega_{1}}\left|\Phi(t) w_{j}(x, t)\right|^{2} d x d t=\frac{1}{2 \pi} \int_{\mathbb{R}} \int_{\omega_{1}}\left|\widehat{\Phi\left(l_{0}\right) w_{j}}\left(x, l_{0}\right)(t)\right|^{2} d x d t \\
\leq & \frac{1}{2 \pi} \int_{\mathbb{R}} \int_{\omega_{1}}\left|\left(1-F_{\lambda}\right) \widehat{\Phi\left(l_{0}\right) w_{j}}\left(x, l_{0}\right)(t)\right|^{2} d x d t+\int_{\mathbb{R}} \int_{\omega_{1}}\left|F_{\lambda} * \Phi(\cdot) w_{j}(x, \cdot)\left(l_{0}\right)\right|^{2} d x d l_{0} .
\end{aligned}
$$

The first term in the right hand side of (47) reads

$$
\begin{aligned}
& \frac{1}{2 \pi} \int_{\mathbb{R}} \int_{\omega_{1}}\left|\left(1-F_{\lambda}\right) \widehat{\Phi\left(\bar{l}_{0}\right) w_{j}}\left(x, l_{0}\right)(t)\right|^{2} d x d t \\
& =\frac{1}{2 \pi} \int_{\mathbb{R}} \int_{\omega_{1}}\left(1-e^{-\left(\frac{t}{\lambda}\right)^{2}}\right)^{2}\left|\widehat{\Phi\left(l_{0}\right) w_{j}}\left(x, l_{0}\right)(t)\right|^{2} d x d t \\
& \leq \frac{1}{\pi \lambda^{2}} \int_{\mathbb{R}} \int_{\omega_{1}}\left|t \Phi{\widehat{\left(l_{0}\right) w_{j}}}_{j}\left(x, l_{0}\right)(t)\right|^{2} d x d t \\
& \leq \frac{2}{\lambda^{2}} \int_{\mathbb{R}} \int_{\omega_{1}}\left|\Phi^{\prime}\left(l_{0}\right) w_{j}\left(x, l_{0}\right)+\Phi\left(l_{0}\right) \partial_{l_{0}} w_{j}\left(x, l_{0}\right)\right|^{2} d x d l_{0}
\end{aligned}
$$




$$
\begin{aligned}
& \leq \frac{4}{\lambda^{2}} \int_{\mathbb{R}} \int_{\omega_{1}}\left(\left|\Phi^{\prime}\left(l_{0}\right) w_{j}\left(x, l_{0}\right)\right|^{2}+\left.\Phi\left(l_{0}\right) \partial_{l_{0}} w_{j}\left(x, l_{0}\right)\right|^{2}\right) d x d l_{0} \\
& \leq \frac{4}{\lambda^{2}}\left[\left(\frac{2}{L}\right)^{2} \int_{K_{0}} \int_{\omega_{1}}\left|w_{j}\left(x, l_{0}\right)\right|^{2} d x d l_{0}+\int_{0}^{L} \int_{\omega_{1}}\left|\partial_{l_{0}} w_{j}\left(x, l_{0}\right)\right|^{2} d x d l_{0}\right] \\
& \leq \frac{4}{\lambda^{2}}\left[\left(\frac{1}{4 A T}\right)^{2} \int_{K_{0}} \int_{\omega_{1}}\left|w_{j}\left(x, l_{0}\right)\right|^{2} d x d l_{0}+\int_{0}^{8 A T} \int_{\omega_{1}}\left|\partial_{l_{0}} w_{j}\left(x, l_{0}\right)\right|^{2} d x d l_{0}\right] .
\end{aligned}
$$

Let

$$
W_{j, \lambda}\left(x, l_{0}\right) \triangleq W_{j}(x, 0)=\int_{\mathbb{R}} F_{\lambda}\left(l_{0}-l\right) \Phi(l) w_{j}(x, l) d l=F_{\lambda} * \Phi(\cdot) w_{j}(x, \cdot)\left(l_{0}\right) .
$$

By applying the Cauchy integral formula, for $\rho \in(0, T-\epsilon)$ and by setting $z=\kappa+\rho e^{i \phi}$, we have that

$$
\begin{aligned}
& W_{j, \lambda}(x, \kappa)=\frac{1}{2 \pi i} \int_{|z-\kappa|=\rho} \frac{W_{j, \lambda}(x, z)}{z-\kappa} d z=\frac{1}{2 \pi i} \int_{0}^{2 \pi} W_{j, \lambda}\left(x, \kappa+\rho e^{i \phi}\right) d \phi \\
& =\frac{1}{2 \pi i(T-\epsilon)} \int_{0}^{T-\epsilon} \int_{0}^{2 \pi} W_{j, \lambda}\left(x, \kappa+\rho e^{i \phi}\right) d \phi d \rho \\
& =\frac{1}{2 \pi i(T-\epsilon)} \int_{-T+\epsilon}^{T-\epsilon} \int_{-\sqrt{(T-\epsilon)^{2}-l_{0}^{2}}}^{\sqrt{(T-\epsilon)^{2}-l_{0}^{2}}} W_{j, \lambda}\left(x, l_{0}+i s\right)\left|J\left(l_{0}, s\right)\right| d s d l_{0} \\
& =\frac{1}{2 \pi i(T-\epsilon)} \int_{-T+\epsilon}^{T-\epsilon} \int_{-\sqrt{(T-\epsilon)^{2}-l_{0}^{2}}}^{\sqrt{(T-\epsilon)^{2}-l_{0}^{2}}} W_{j}(x, s) d s d l_{0} .
\end{aligned}
$$

Thus,

$$
\left|W_{j, \lambda}(x, \kappa)\right|^{2}=\frac{1}{\pi^{2}} \int_{-T+\epsilon}^{T-\epsilon} \int_{-T+\epsilon}^{T-\epsilon}\left|W_{j}(x, s)\right|^{2} d s d l_{0} .
$$

Integrating (51) with respect to $x$ over $\omega_{1}$ and with respect to $\kappa$ over $\left[-\frac{L}{2}, \frac{L}{2}\right]$, we get that

$$
\begin{aligned}
& \int_{-4 A T}^{4 A T} \int_{\omega_{1}}\left|W_{j, \lambda}(x, \kappa)\right|^{2} d x d \kappa \\
& \leq \frac{1}{\pi(1-\epsilon)^{2}} \int_{-4 A T}^{4 A T} \int_{-T+\epsilon}^{T-\epsilon}\left(\int_{-T+\epsilon}^{T-\epsilon} \int_{\omega_{1}}\left|W_{j}(x, s)\right|^{2} d x d s\right) d l_{0} d \kappa \\
& \leq \frac{16 A T(T-\epsilon)}{\pi^{2}} \int_{-T+\epsilon}^{T-\epsilon} \int_{\omega_{1}}\left|W_{j}(x, s)\right|^{2} d x d s .
\end{aligned}
$$


Substituting (48), (52) into (47) and noting that $T=1$, we find that

$$
\begin{aligned}
& \left\|\Phi w_{j}\right\|_{L^{2}\left(\omega_{1} \times(-4 A, 4 A)\right)}^{2} \\
& \leq \frac{4}{\lambda^{2}}\left[\frac{1}{16 A^{2}} \int_{K_{0}} \int_{\omega_{1}}\left|w_{j}\left(x, l_{0}\right)\right|^{2} d x d l_{0}+\int_{-4 A}^{4 A} \int_{\omega_{1}}\left|\partial_{l_{0}} w_{j}\left(x, l_{0}\right)\right|^{2} d x d l_{0}\right] \\
& \quad+\frac{16 A}{\pi^{2}} \int_{-1+\epsilon}^{1-\epsilon} \int_{\omega_{1}}\left|W_{j}(x, s)\right|^{2} d x d s .
\end{aligned}
$$

Similarly, we can obtain that

$$
\begin{aligned}
& \left\|\nabla\left(\Phi w_{j}\right)\right\|_{L^{2}\left(\omega_{1} \times(-4 A, 4 A)\right)}^{2} \\
& \leq \frac{4}{\lambda^{2}}\left[\frac{1}{16 A^{2}} \int_{K_{0}} \int_{\omega_{1}}\left|\nabla w_{j}\left(x, l_{0}\right)\right|^{2} d x d l_{0}+\int_{-4 A}^{4 A} \int_{\omega_{1}}\left|\partial_{l_{0}} \nabla w_{j}\left(x, l_{0}\right)\right|^{2} d x d l_{0}\right] \\
& \quad+\frac{16 A}{\pi^{2}} \int_{-1+\epsilon}^{1-\epsilon} \int_{\omega_{1}}\left|\nabla W_{j}(x, s)\right|^{2} d x d s .
\end{aligned}
$$

Let $\sigma=\frac{\lambda^{2}}{2 \tau \mu_{3}}$ and $C_{2}=\max \left\{|\nabla \chi|^{2},|\Delta \chi|^{2}\right\}$ such that

$$
e^{\sigma\left(2 \mu_{3}-\left(2-\delta_{2}\right) \mu_{2}\right)} e^{\frac{1-A^{2}}{2}} \leq 1 .
$$

From (45), (47), (53) and (54), we have

$$
\begin{aligned}
& \sum_{j=1}^{2}\left\|a_{j j}-\tilde{a}_{j j}\right\|_{L^{2}(\Omega)}^{2} \\
& \leq C\left\{\frac { 4 } { \lambda ^ { 2 } } \left[\frac{1}{16 A^{2}} \int_{K_{0}} \int_{\omega_{1}} \sum_{j=1}^{2}\left(\left|w_{j}\left(x, l_{0}\right)\right|^{2}+\left|\nabla w_{j}\left(x, l_{0}\right)\right|^{2}\right) d x d l_{0}\right.\right. \\
& \left.+\int_{-4 A}^{4 A} \int_{\omega_{1}} \sum_{j=1}^{2}\left(\left|\partial_{l_{0}} w_{j}\left(x, l_{0}\right)\right|^{2}+\left|\partial_{l_{0}} \nabla w_{j}\left(x, l_{0}\right)\right|^{2}\right) d x d l_{0}\right] \\
& +\frac{1}{\pi^{2}}\left(1+\frac{1}{1-\epsilon}\right)^{2} C_{0} \frac{256 A^{2} \lambda^{2}}{\pi \sigma}\left[\left(\frac{1}{32 A^{2}} e^{\sigma\left(2 \mu_{3}-\left(2-\delta_{2}\right) \mu_{2}\right)} e^{\frac{1-A^{2}}{2} \lambda^{2}}\right.\right. \\
& \left.+e^{-2 \sigma \tau \mu_{3}} e^{\frac{\lambda^{2}}{2}} \max \left\{|\nabla \chi|^{2},|\Delta \chi|^{2}\right\}\right) \mathbf{C} \\
& \left.\left.+\frac{\sigma^{4}}{2} e^{\sigma\left(2 \mu_{3}-\left(2-\delta_{2}\right) \mu_{2}\right)} e^{\frac{\lambda^{2}}{2}}|| w_{1} \|_{L^{2}\left(-4 A, 4 A ; H^{1}(\omega)\right)}^{2}\right]\right\} \\
& \leq C\left\{\left[\frac{8}{\lambda^{2}}+C_{0} \frac{256 A^{2} \lambda^{2}}{\pi^{3} \sigma} e^{-2 \sigma \tau \mu_{3}}\left(\frac{1}{32 A^{2}} e^{-\frac{A^{2}-1}{2} \lambda^{2}}+C_{2} e^{\frac{\lambda^{2}}{2}}\right)\right] \mathbf{C}\right.
\end{aligned}
$$




$$
\begin{aligned}
+ & \left.C_{0} \frac{128 A^{2} \lambda^{2} \sigma^{3}}{\pi^{3}} e^{\sigma\left(2 \mu_{3}-\left(2-\delta_{2}\right) \mu_{2}\right)} e^{\frac{\lambda^{2}}{2}}\left\|w_{1}\right\|_{L^{2}\left(-4 A, 4 A ; H^{1}(\omega)\right)}^{2}\right\} \\
\leq C & \left\{\left[\frac{8}{\lambda^{2}}+C_{0} \frac{512 A^{2} \tau \mu_{3}}{\pi^{3}}\left(\frac{1}{32 A^{2}} e^{-\frac{A^{2}+1}{2} \lambda^{2}}+C_{2} e^{-\frac{\lambda^{2}}{2}}\right)\right] \mathbf{C}\right. \\
& \left.+C_{0} \frac{16 \tau^{3} \mu_{3}^{3} A^{2}}{\pi^{3} \lambda^{4}} e^{\left(\frac{2 \mu_{3}-\left(2-\delta_{2}\right) \mu_{2}}{2 \tau \mu_{3}}+\frac{1}{2}\right) \lambda^{2}}\left\|w_{1}\right\|_{L^{2}\left(-4 A, 4 A ; H^{1}(\omega)\right)}^{2}\right\} .
\end{aligned}
$$

Let $\lambda \geq \lambda_{0}$ be such that

$$
\sum_{j=1}^{2}\left\|a_{j}-\tilde{a}_{j}\right\|_{L^{2}(\Omega)}^{2} \leq \frac{C_{3}}{\lambda^{2}} \mathbf{C}+e^{C_{4} \lambda^{2}}\left\|w_{1}\right\|_{L^{2}\left(-T, T ; H^{1}(\omega)\right)}^{2}
$$

where $C_{3}$ and $C_{4}$ are two constants independent of $\lambda$. Taking

$$
\lambda=\max \left\{\lambda_{0},\left(\frac{|\ln |\left|w_{1}\right|_{L^{2}\left(-T, T ; H^{1}(\omega)\right)} \mid}{C_{4}}\right)^{\frac{1}{2}}\right\}
$$

If $\left\|w_{1}\right\|_{L^{2}\left(0, T ; H^{1}(\omega)\right)}$ is small enough, then

$$
\begin{aligned}
\sum_{j=1}^{2}\left\|a_{j j}-\tilde{a}_{j j}\right\|_{L^{2}(\Omega)}^{2} & \leq \frac{C_{3} C_{4}}{\left.|\ln |\left|w_{1}\right|\right|_{L^{2}\left(-T, T ; H^{1}(\omega)\right)} \mid} \mathbf{C}+\left\|w_{1}\right\|_{L^{2}\left(-T, T ; H^{1}(\omega)\right)} \\
& \leq C\left(\left.|\ln |\left|w_{1}\right|_{L^{2}\left(-T, T ; H^{1}(\omega)\right)}\right|^{-1} \mathbf{C}+\left\|w_{1}\right\|_{L^{2}\left(-T, T ; H^{1}(\omega)\right)}\right) \\
& \leq C\left(\left.|\ln |\left|w_{1}\right|_{L^{2}\left(0, T ; H^{1}(\omega)\right)}\right|^{-1} \mathbf{C}+\left\|w_{1}\right\|_{L^{2}\left(0, T ; H^{1}(\omega)\right)}\right) .
\end{aligned}
$$

Otherwise, there exists a constant $m>0$ such that $\left\|w_{1}\right\|_{L^{2}\left(-T, T ; H^{1}(\omega)\right)} \geq m$. Thus, by (30) we have

$$
\sum_{j=1}^{2}\left\|a_{j j}-\tilde{a}_{j j}\right\|_{L^{2}(\Omega)}^{2} \leq \mathbf{C}=\frac{\mathbf{C}}{m} m \leq C\left\|w_{1}\right\|_{L^{2}\left(-T, T ; H^{1}(\omega)\right)} \leq C\left\|w_{1}\right\|_{L^{2}\left(0, T ; H^{1}(\omega)\right)} .
$$

\section{acknowledgement}

The first author thanks the support of the National Natural Science Foundation of China (No. 11501086), the Fundamental Research Funds for the Central Universities (No. ZYGX2016J137) and the Science Strength Promotion Programme of UESTC. The second author is supported by Grant-in-Aid for Scientific Research (S) 15H05740 and A3 Foresight Program Modeling and Computation of Applied Inverse Problems of Japan Society for the Promotion of Science, and the RUDN University Program 5-100. 


\section{References}

[1] A. D. Bandrauk. Molecules in Laser Fields. M. Dekker, 1994.

[2] L. Baudouin and J. P. Puel. Uniqueness and stability in an inverse problem for the Schrödinger equation. Inverse Problems. 18(2007),1537.

[3] L. Baudouin and A. Mercado. An inverse problem for Schrodinger equations with discontinuous main coefficient. Appl. Anal. 87(2008), 1145-1165.

[4] M. Bellassoued, Y. Kian and E. Soccorsi. An inverse stability result for non-compactly supported potentials by one arbitrary lateral Neumann observation. J. Differential Equations 260(2016), 7535-7562.

[5] M. Bellassoued and M. Choulli. Logarithmic stability in the dynamical inverse problem for the Schrödinger equation by arbitrary boundary observation. J. Math. Pures Appl. 91(2009), 233-255.

[6] A. Benabdallah, M. Cristofol, P. Gaitan,M. Yamamoto. Inverse problem for a parabolic system with two components by measurements of one component. Appl. Anal. $\mathbf{8 8}$ (2009), 683-709.

[7] L. Cardoulis. An inverse problem for a time-dependent Schrödinger operator in an unbounded strip. C. R. Math. Acad. Sci. Paris 350(2012), 891-896.

[8] L. Cardoulis and P. Gaitan. Simultaneous identification of the diffusion coefficient and the potential for the Schrödinger operator with only one observation. Inverse Problems 26(2010), 035012.

[9] J.-M. Delort. F.B.I. transformation. Springer-Verlag, Berlin, 1992.

[10] L. Deng. An inverse problem for the Schrödinger equation with variable coefficients and lower order terms. J. Math. Anal. Appl. 427(2015), 930-940.

[11] A. V. Fursikov and O. Yu. Imanuvilov. Controllability of evolution equations. Seoul National University, Research Institute of Mathematics, Global Analysis Research Center, Seoul, 1996.

[12] A. Giusti-Suzor and F. H. Mies. Vibrational trapping and suppression of dissociation in intense laser fields. Phys. Rev. Lett. 68(1992), 3869-3872.

[13] M. González-Burgos and L. de Teresa. Controllability results for cascade systems of $m$ coupled parabolic PDEs by one control force. Port. Math. 67(2010), 91-113. 
[14] Y. Kian, Q. S. Phan and E. Soccorsi, A Carleman estimate for infinite cyclindrical quantum domains and the application to inverse problems. Inverse Problems 30(2014), 055016 .

[15] I. Lasiecka, R. Triggiani and X. Zhang. Global uniqueness, observability and stabilization of nonconservative Schrödinger equations via pointwise Carleman estimates. I. $H^{1}(\Omega)$-estimates. J. Inverse Ill-Posed Probl. 12(2004), 43-123.

[16] I. Lasiecka, R. Triggiani and X. Zhang. Global uniqueness, observability and stabilization of nonconservative Schrödinger equations via pointwise Carleman estimates. II. $L^{2}(\Omega)$-estimates. J. Inverse Ill-Posed Probl. 12 (2004), 183-231.

[17] A. Mercado, A. Osses and L. Rosier. Inverse problems for the Schrödinger equation via Carleman inequalities with degenerate weights. Inverse Problems 24(2008), 015017.

[18] K. D. Phung. Observability and control of Schrödinger equations. SIAM J. Control Optim. 40(2001), 211-230.

[19] G. Yuan and M. Yamamoto. Carleman estimates for the Schrödinger equation and applications to an inverse problem and an observability inequality. Chin. Ann. Math. Ser. B 31(2010), 555-578. 